\title{
Geomagnetic jerks and rapid hydromagnetic waves focusing at Earth's core surface
}

\author{
Aubert, Julien; Finlay, Christopher C.
}

Published in:

Nature Geoscience

Link to article, DOI:

$10.1038 / \mathrm{s} 41561-019-0355-1$

Publication date:

2019

Document Version

Peer reviewed version

Link back to DTU Orbit

Citation (APA):

Aubert, J., \& Finlay, C. C. (2019). Geomagnetic jerks and rapid hydromagnetic waves focusing at Earth's core surface. Nature Geoscience, 12(5), 393-398. https://doi.org/10.1038/s41561-019-0355-1

\section{General rights}

Copyright and moral rights for the publications made accessible in the public portal are retained by the authors and/or other copyright owners and it is a condition of accessing publications that users recognise and abide by the legal requirements associated with these rights.

- Users may download and print one copy of any publication from the public portal for the purpose of private study or research.

- You may not further distribute the material or use it for any profit-making activity or commercial gain

- You may freely distribute the URL identifying the publication in the public portal

If you believe that this document breaches copyright please contact us providing details, and we will remove access to the work immediately and investigate your claim 


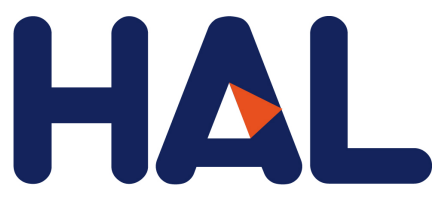

archives-ouvertes

\title{
Geomagnetic jerks and rapid hydromagnetic waves focusing at Earth's core surface
}

\author{
Julien Aubert, Christopher Finlay
}

\section{To cite this version:}

Julien Aubert, Christopher Finlay. Geomagnetic jerks and rapid hydromagnetic waves focusing at Earth's core surface. Nature Geoscience, Nature Publishing Group, 2019, 12 (5), pp.393-398. 10.1038/s41561-019-0355-1 . hal-02129842

\section{HAL Id: hal-02129842 \\ https://hal.archives-ouvertes.fr/hal-02129842}

Submitted on 15 May 2019

HAL is a multi-disciplinary open access archive for the deposit and dissemination of scientific research documents, whether they are published or not. The documents may come from teaching and research institutions in France or abroad, or from public or private research centers.
L'archive ouverte pluridisciplinaire HAL, est destinée au dépôt et à la diffusion de documents scientifiques de niveau recherche, publiés ou non, émanant des établissements d'enseignement et de recherche français ou étrangers, des laboratoires publics ou privés. 


\title{
Geomagnetic jerks and rapid hydromagnetic waves focusing at Earth's core surface
}

\author{
Julien Aubert $^{1 *}$ and Christopher C. Finlay ${ }^{2}$
}

${ }_{4}{ }^{1}$ Institut de Physique du Globe de Paris, Sorbonne Paris Cité, Université Paris Diderot, CNRS

5 UMR 7154 CNRS, F-75005 Paris, France. ${ }^{(*)}$ : aubert@ipgp.fr

${ }_{6}{ }^{2}$ Division of Geomagnetism, National Space Institute, Technical University of Denmark, Elektro7 vej, 2800 Kgs. Lyngby, Denmark

Geomagnetic jerks are abrupt changes in the second time derivative - the secular accelerationof Earth's magnetic field that punctuate ground observatory records. They are presently the major obstacle to the prediction of geomagnetic field behaviour years to decades ahead. Recent jerks have been linked to short-lived, temporally alternating and equatorially localised pulses of secular acceleration observed in satellite data, associated with rapidly alternating flows at Earth's core surface. The dynamical origin of jerks has been unclear but can now be investigated in numerical models of the geodynamo that realistically simulate the interaction between slow core convection and rapid hydromagnetic waves. Using one such model, here we show that the observed jerk patterns can be explained by the arrival of localised Alfvén wave packets radiated from sudden buoyancy releases inside the core. As they reach the core surface, the waves focus their energy towards the equatorial plane and along lines of strong magnetic flux, creating sharp interannual changes in core flow and producing geomagnetic jerks through the induced variations in field acceleration. The ability to numerically repro- 


\section{duce jerks offers a new way to probe the physical properties of Earth's deep interior.}

The geomagnetic field displays temporal variations on a broad range of time scales. Through a self-sustained dynamo process (the geodynamo), slow convective motion in Earth's electrically conducting and liquid core is believed to maintain the field and drive its changes over centuries and longer. At the other end of the range, geomagnetic jerks with typical time scales a few years or less ${ }^{1}$ represent the fastest observed features of the internally-generated field. They were initially identified as 'V-shaped' patterns (see examples in Fig. 1a) in time series of the magnetic field rate-of-change at ground observatories ${ }^{2,3}$ (the secular variation), indicating an abrupt change in the field acceleration amidst periods where this acceleration is otherwise approximately constant. Explaining the time scale disparity between rapid jerks and slow convection is a theoretical challenge that has recently spurred significant progress, both in observational geomagnetism and in numerical geodynamo simulations. In combination with an improving network of ground observatories, satellite magnetic field observations now provide a global and continuous view of the geomagnetic secular acceleration over the past two decades ${ }^{4,5}$, with horizontal spatial resolution of approximately $2000 \mathrm{~km}$ at the core surface (spherical harmonic degree 9) and temporal resolution down to about a year on the largest length scales. This has dramatically enhanced our empirical knowledge of jerks, most notably by revealing ${ }^{6-9}$ their links to short-lived, temporally alternating pulses of geomagnetic acceleration at the surface of the Earth (Fig. 1c,e), that at the core surface are most prominent at low latitudes and localised in longitude (Fig. 2a,b). In the wake of these results, the earlier historical jerks of the twentieth century have also been linked to considerably less resolved, but similarly alternating pulses with low-latitude foci ${ }^{10}$. It has long been suspected that 
jerks could somehow represent the signature of hydromagnetic waves ${ }^{11}$. This prompted an earlier explanation ${ }^{12}$ for jerks in terms of time-varying zonal flows that are kinematically consistent with torsional Alfvén waves occurring between concentric, magnetically-coupled, axial cylinders in the core. Though torsional waves have later been successfully identified in Earth's core ${ }^{13}$ and in selfconsistent numerical simulations of the geodynamo ${ }^{14-17}$, they have however been found to occur at interannual periods shorter than earlier decadal estimates, and with an amplitude that is too weak to account for the geomagnetic secular acceleration signal associated with jerks ${ }^{17,18}$. Furthermore, the complex patterns of magnetic acceleration found in satellite observations require localised (i.e. non-axisymmetric), rapidly alternating flows beneath the core surface ${ }^{5,19-21}$. These discount an explanation in terms of torsional waves, but provide valuable new constraints on the rapid dynamics taking place in Earth's core.

\section{Rapid hydromagnetic waves in advanced numerical geodynamo simulations}

Numerical simulations of convective core magnetohydrodynamics have been successful at describing the detailed morphology of the geomagnetic field ${ }^{22,23}$, its temporal variations and the underlying core flows ${ }^{24}$. To achieve this, the magnetic Reynolds number comparing the magnetic diffusion and convective core overturn time scales $\tau_{\eta}$ and $\tau_{U}$ (see definitions in Methods) needs to be $R m=\tau_{\eta} / \tau_{U} \approx 1000$, such that a realistic $\tau_{U} \approx 100 \mathrm{yr}$ is achieved when adopting an Earth-like value $\tau_{\eta} \approx 10^{5} \mathrm{yr}$ as the fundamental time scale for casting the dimensionless model results back to the dimensional world (Methods). However, most existing simulations remain unrealistic when considering time scales significantly shorter than $\tau_{U}$. In Earth's core, the dynamics of geomagnetic 
jerks will involve strong rotational constraints from the Coriolis force, because the jerk's interannual time scale is much longer than Earth's planetary rotation period $2 \pi \tau_{\Omega}=1$ day. Jerk dynamics will also be affected by hydromagnetic waves given the proximity of the Alfvén time ${ }^{13} \tau_{A} \approx 2 \mathrm{yr}$. However, because of computational limitations ${ }^{16,25}$, and despite continuous advances ${ }^{14-16,26,27}$, neither of these processes are correctly rendered in standard simulations where $2 \pi \tau_{\Omega}$ and $\tau_{A}$ remain much too long, and not sufficiently separated from $\tau_{U}$. For instance, in our previous Coupled Earth dynamo model ${ }^{24} 2 \pi \tau_{\Omega} \approx 10 \mathrm{yr}$ and $\tau_{A} \approx \tau_{U} \approx 100 \mathrm{yr}$. In order to remedy these problems, we have recently introduced a suite of numerical simulations ${ }^{17,25}$ following a well-defined path through control parameter space that connects the original coupled Earth model to Earth's core conditions. Our new simulations (Methods and Supplementary Table 1) involve a reasonably accurate large-scale approximation that enables the exploration of parameters significantly beyond current computational limits for direct numerical simulations. Along this path, the Earth-like field morphology and kinematics of the coupled Earth model used as starting point are preserved, as are the values of $\tau_{U}$ and $\tau_{\eta}$, but the dynamics gradually evolves as $2 \pi \tau_{\Omega}$ and $\tau_{A}$ decrease to become realistic and increasingly separated from $\tau_{U}$ and $\tau_{\eta}$. An asymptotic regime of strong rotational and magnetic control pertaining to Earth's core conditions is reached ${ }^{17}$ at path positions beyond $30 \%$. In addition to the slow background convection at time scale $\tau_{U}$ that is present throughout the path, models in this regime additionally feature rapid magneto-inertial wave dynamics at time scale $\tau_{A}$. This dynamics includes geostrophic torsional Alfvén waves of weak amplitude, and also non-axisymmetric, quasi-geostrophic Alfvén waves ${ }^{17}$. The relevance of the latter waves, that were previously unexpected ${ }^{28}$, has only recently been released ${ }^{17,29}$. They offer a promising explana- 
tion to jerks since they have been linked ${ }^{17}$ to the occurrence of intermittent magnetic acceleration pulses occurring at low latitudes. In our most advanced Midpath model (50\% of the path, Methods and Supplementary Table 1), the Alfvén time scale value $\tau_{A}=14.3 \mathrm{yr}$ implies that the waves have interannual periods at wavelengths a fraction of the core size, well separated from convective processes. Since $\tau_{A} \gg 2 \pi \tau_{\Omega}=0.19 \mathrm{yr}$, such periods are also well within the rotationally-dominated range where the Coriolis force plays a crucial role. Finally, a high ratio $\tau_{\eta} / \tau_{A} \approx 10^{4}$ of the magnetic diffusion and Alfvén time scales indicates that wave attenuation will be weak on large length scales.

\section{Observed and simulated geomagnetic jerks}

Short-lived, intermittent pulses in the magnetic acceleration energy at Earth's surface (Fig. 1d) are observed in numerical simulations throughout the parameter space path, but migrate to low latitudes (Fig. 1f) only once the model conditions enter the rapid rotation regime ${ }^{17}$. In order to highlight the link between such pulses and jerks, and to facilitate comparison with geomagnetic field models of limited temporal resolution (Fig. 1c,e), we define jerk energy (Methods) as the mean-squared difference between time averages of Earth's surface magnetic acceleration taken within two consecutive and non-overlapping 3-year time windows. With this definition, the timing of jerks in the simulation (Fig. 1d) can be properly defined from jerk energy pulses, and is found to either shortly precede or follow that of magnetic acceleration pulses, as observed with well-documented recent geomagnetic jerks ${ }^{6}$ (Fig. 1c). The intensity and duration of the events also match rather well the observations. Abrupt slope changes in the magnetic variation time se- 
ries are observed at specific locations (Fig. 1b), with approximately constant acceleration away from the event, similar to the classic 'V-shaped' jerk signatures seen at ground observatories ${ }^{1-3}$ (Fig. 1a). Maps of the radial magnetic acceleration before and after the events feature alternating patterns (Fig. 1f) and indicate that simulated jerks are often visible over a large area (from America to Indonesia for the event shown here) at low and mid-latitudes, comparable to observations of the well-characterised 2007.5 geomagnetic jerk (Fig. 1e) and to a number of earlier events ${ }^{30,31}$. Descending to the core surface (Fig. 2c,d), the corresponding structures are series of intense and oppositely-signed patches of radial magnetic acceleration generated close to the equator and in a narrow longitudinal band, beneath westward-drifting patches of intense radial magnetic flux (see Fig. 3e) localised in the Atlantic hemisphere ${ }^{17,24}$. The field acceleration patches alternate rapidly in time for a few years (Supplementary Movie 1 from time $-10 \mathrm{yr}$ to $10 \mathrm{yr}$ ) before fading away. The spatially localised morphology, interannual alternation time scale and the amplitude (approximately 2,000 nT.yr ${ }^{-2}$ up to degree 9) of the simulation output reproduce well the core surface signature of geomagnetic jerks ${ }^{5,8}$ (Fig. 2a,b, see the events in 2007.5, 2011 and 2014.5).

\section{The origin of geomagnetic jerks and the role of hydromagnetic waves}

At the large scales accessible to observations (spherical harmonic degree up to 9), and in the rapid rotation regime, the magnetic acceleration pulses in the simulations result from the action of accelerating azimuthal core surface flows ${ }^{17}$ rather than from diffusive processes related to flux expulsion that are common at the start of the parameter space path. In our Midpath model sequence, a localised, intense and temporally alternating pulse of azimuthal flow acceleration is 
indeed observed in the vicinity of the jerk time (Fig 2e,f, Supplementary Movie 2), resembling the localised alternating flows that have been inferred from geomagnetic variations ${ }^{5}$ associated with the 2007.5 geomagnetic jerk. The source of this perturbation can be traced back to a sudden buoyancy release from an isolated density anomaly at mid-depth in the core, 25 years before the event (Supplementary Movies 3,4). This release triggers strong azimuthal fluid flow accelerations that are entrained within the associated convective plume towards the core surface. The plume stalls at a cylindrical radius $s_{c} \approx 2950 \mathrm{~km}$ (Supplementary Movie 3, Supplementary Fig. 1) where its decreasing radial velocity is overcome by the global westward drift. At cylindrical radii above $s_{c}$, we identify quasi-geostrophic Alfvén waves ${ }^{17,29}$ through the adherence of their trajectories to propagation at the locally variable, theoretical Alfvén speed and the deviation of their paths from that of material upwellings (Methods and Supplementary Fig. 1). In the upper outer core, material upwelling is indeed much slower than Alfvén waves, or even directed inwards. The perturbation energy however propagates further towards the core surface in well-defined, azimuthally extended, alternating wavefronts (Fig. 3a,b, Supplementary Movie 4) of columnar structure characteristic of rotationally-dominated dynamics ${ }^{17}$. The waves have a radial wavelength $d \approx D / 4$ that is in line with the size of the density anomaly that initiated the event. Their energy becomes spatially concentrated as they approach the core-mantle boundary (Fig. 3c), yielding the intense, localised and temporally alternating surface flow acceleration signature (Fig. 2e,f) that causes the jerk, on a time scale comparable to the Alfvén wave period for these structures, $\sqrt{3} \tau_{A} d / D \approx 6 \mathrm{yr}$. The energy concentration mechanism can be understood by noting that quasi-geostrophic Alfvén wavefronts are both guided along, and bounded by a strongly heterogeneous distribution of magnetic 
field lines ${ }^{17}$. Beneath the jerk location, these field lines are arranged in an approximately axiallyinvariant funnel-like structure (Fig. 3d) that is shaped by the slow convection and remains approximately static during the event. This causes the waves to be longitudinally focused towards a pair of intense radial magnetic flux patches (see arrows in Fig. 3e) at the core surface. At the same time, latitudinal focusing towards the equator occurs because of the effect of the spherical core-mantle boundary on flow columns that tend to preserve their angular momentum as their height decreases (Fig. 3b, Supplementary Video 4). Finally, the wave speed decreases close to the core-mantle boundary (see curved green tracks in Supplementary Fig. 1) because the magnetic field is weaker at the surface than at depth ${ }^{25}$. To preserve the energy flux, the amplitude of wavefronts increases, and preservation of the wave period also implies a reduction of the radial wavelength (Fig. 3c), similarly to a shoaling process for water waves ${ }^{32}$. This three-dimensional energy focusing mechanism is crucial in amplifying the weak quasi-geostrophic Alfvén waves so as to produce localised and temporally alternating disturbances in the core surface flow acceleration that are significant enough to cause jerks.

\section{Implications for geomagnetism and global geodynamics}

Since our models are in the dynamical regime of rapid rotation and strong magnetic control relevant to Earth's core ${ }^{17,25}$, their results can be extrapolated to natural conditions. From the mechanism described here, the duration and alternation time scale of jerk events are expected to scale with the Alfvén time $\tau_{A}$, which is about 7 times shorter in Earth's core ${ }^{13}$ than in our Midpath simulation (Supplementary Table 1). Yet the observed geomagnetic acceleration changes are only two 
to three times faster than those simulated by the Midpath model (Fig. 2). This discrepancy is likely related to the limited temporal resolution of present geomagnetic field models, which prevents the true, potentially sub-annual ${ }^{33}$ variations associated with jerks from being retrieved. In the upcoming years, further insight will be obtained from jerk events that will be imaged with improved resolution using data collected by the Swarm satellite mission. As we move along the parameter space path, our models also indicate that energetic jerks occur more frequently (Fig. 4a). It is possible to construct statistical relationships between jerk energy and recurrence time (Fig. 4b), and derive a scaling relationship for the evolution along the path of jerk energy at a given recurrence time (Supplementary Fig. 2) in reasonable agreement with a theoretical prediction (Methods). The extrapolation of this relationship to the end of the path (Fig. 4b) also agrees with the observed subdecadal to decadal jerk recurrence rates observed in the geomagnetic field ${ }^{1,5}$. Jerk energy is also found to decrease with increasing lower mantle conductance, because of the associated additional Ohmic losses, and with increasing levels of upper outer core stratification (Supplementary Fig. 3). This latter effect is due to changes in the geometry and amplitude of the background magnetic field rather than to the wave mechanism itself, which is not sensitive to stratification. Finally, examining simulated records of the length of day (Methods, Supplementary Fig. 4) in the vicinity of jerk events, we also observe signatures of the wave's arrival at the core surface. Rapid inflexions in the rate of change of the length-of-day similar to those observed for Earth ${ }^{31,34}$ are caused by pulses in the acceleration of the electromagnetic torque felt by the mantle. All these results highlight the potential importance of the numerical reproduction of jerks, as it may lead to an improved geomagnetic ${ }^{35,36}$ and geodetic ${ }^{37}$ sounding of important, but poorly known physical properties such 
as the lower mantle electrical conductivity and upper outer core thermal conductivity.

The integration of geomagnetic data into numerical geodynamo simulations through data assimilation has significantly advanced in the recent past ${ }^{38}$, leading to inferences of the dynamical internal structure of the geodynamo and to predictions of the future geomagnetic field evolution ${ }^{39}$ that have been integrated within the latest iteration ${ }^{40,41}$ of the International Geomagnetic Reference Field (IGRF). At interannual to decadal time scales, the accuracy of such predictions is currently hampered by the underlying dynamical model, which is located at the start of the parameter space path and hence does not account for wave dynamics. The availability of advanced numerical dynamo simulations that produce realistic rapid dynamics and jerks will significantly improve the quality of the prior information on which the predictions are based (in particular regarding the time-dependence of the field), with subsequent gains in their accuracy. 


\section{Methods}

Model description. The full description of our numerical models can be found in refs. ${ }^{17,25}$. We solve for Boussinesq convection, thermochemical density anomaly transport and magnetic induction in the magnetohydrodynamic approximation within an electrically conducting and rotating spherical fluid shell of thickness $D=r_{o}-r_{i}$ representing the outer core, with $r_{i} / r_{o}=0.35$ as in the Earth. Our unknowns are the velocity field $\mathbf{u}$, magnetic field $\mathbf{B}$ and density anomaly field $C$, and we analyse the magnetic variation $\partial \mathbf{B} / \partial t$, magnetic acceleration $\partial^{2} \mathbf{B} / \partial t^{2}$ and the flow acceleration $\partial \mathbf{u} / \partial t$. The fluid shell is electromagnetically coupled both to a solid inner core of radius $r_{i}$ and to a solid outer shell representing the mantle between radii $r_{o}$ and $1.83 r_{o}$. The inner core and mantle are furthermore coupled together by a gravitational restoring torque. Both the inner core and mantle feature a time-dependent axial differential rotation with respect to the outer core. The three regions are assigned moments of inertia respecting the proportions ${ }^{24}$ relevant to Earth's mantle, inner and outer core, and the ensemble has a constant angular momentum defining the planetary rotation rate $\Omega$.

The mechanical boundary conditions are of the stress-free type at both boundaries. In the low viscosity regime where our models operate, these are undistinguishable from no-slip conditions ${ }^{25}$ while alleviating the need to resolve the viscous boundary layers. Electrically conducting boundary conditions are used at both boundaries. The electrical conductivity of the inner core is set at the same value $\sigma_{c}$ as that of the outer core. The mantle features an electrically conducting region at its base, with thickness $\Delta$ and conductivity $\sigma_{m}$. In our four main model cases (Supplementary Table 1) the dimensionless conductance has been set to a median geophysical estimate ${ }^{42}$ 
$\Sigma=\Delta \sigma_{m} / D \sigma_{c}=10^{-4}$. Two other models (Midpath-I and Midpath-H) explore the end-member values $\Sigma=0$ (insulating mantle) and $\Sigma=10^{-3}$. The thermochemical boundary conditions are of heterogeneous, fixed-flux type. The homogeneous part $F$ of the density anomaly flux is prescribed at the inner boundary. In our four main model cases the homogeneous density anomaly flux vanishes at the outer boundary (neutral buoyancy). A volumetric sink term is then present in the density anomaly transport equation to conserve mass. Within the Boussinesq approximation, this configuration models bottom-driven chemical convection originating from inner core solidification, a fully convective outer core and an exactly adiabatic heat flow at the core-mantle boundary. An additional model (Midpath-S) explores the effect of a possible stratification of the upper outer core ${ }^{43}$ by prescribing an negative (adverse buoyancy) density anomaly flux at the coremantle boundary (see Stratified Core section below). Spatial modulations of the density anomaly fluxes are prescribed at both boundaries ${ }^{17}$, with the same geometry as in the coupled Earth model ${ }^{24}$. These are meant to model a spatially heterogeneous growth of the inner core, and thermal control from the heterogeneous lower mantle.

Model parameters, parameter space path and time scales. The four main control parameters of the model are the flux-based Rayleigh, Ekman, Prandtl and magnetic Prandtl numbers

$$
\begin{aligned}
R a_{F} & =\frac{g_{o} F}{4 \pi \rho \Omega^{3} D^{4}}, \\
E & =\frac{v}{\Omega D^{2}}, \\
P r & =\frac{v}{\kappa}, \\
P m & =\frac{v}{\eta} .
\end{aligned}
$$


Here $g_{o}, \rho, v, \kappa$ and $\eta$ are respectively the gravity at the outer boundary of the model, the fluid density, viscosity, thermo-chemical and magnetic diffusivities $\left(\eta=1 / \mu \sigma_{c}\right.$, with $\mu$ the fluid magnetic permeability). We have recently introduced ${ }^{25}$ the concept of a unidimensional path in parameter space, by showing that the variations in these control parameters that are necessary to bridge the gap between our previous coupled Earth model ${ }^{24}$ and Earth's core conditions can be represented as power laws of a single variable $\epsilon$. Any model along the path is defined using the following rules:

$$
\begin{aligned}
R a_{F} & =\epsilon R a_{F}(\mathrm{CE}), \\
E & =\epsilon E(\mathrm{CE}), \\
P r & =1, \\
P m & =\sqrt{\epsilon} P m(\mathrm{CE}) .
\end{aligned}
$$

Here $R a_{F}(\mathrm{CE})=2.710^{-5}, E(\mathrm{CE})=310^{-5}$ and $P m(\mathrm{CE})=2.5$ are the control parameters of the coupled Earth dynamo model defining the start of the path $(\epsilon=1)$, and we have $\operatorname{shown}^{25}$ that conditions relevant to Earth's core are reached at the end of path defined by $\epsilon=10^{-7}$. Our models are defined in refs. ${ }^{17,25}$ and in Supplementary Table 1 by the values $\epsilon=10^{-2}, 3.3310^{-3}, 10^{-3}$ and $3.3310^{-4}$, respectively corresponding to $29 \%, 35 \%, 43 \%$, and $50 \%$ of the path (the Midpath model). The parameters of the Midpath model are the closest to Earth's core conditions employed to date in a numerical dynamo simulation, at the expense of a large scale approximation (see Numerical Implementation section below).

The model outputs follow scaling laws ${ }^{25}$ depending on $\epsilon$ that also closely approach the conditions expected in Earth's core as we progress along the path (Supplementary Table 1). Once the 
magnetic diffusion time $\tau_{\eta}=D^{2} / \eta$ is set to an Earth-like value (see Rescaling section below), the end of path simultaneously matches the Earth's core rotational time $\tau_{\Omega}=1 / \Omega$, convective overturn time $\tau_{U}=D / U$, and Alfvén time $\tau_{A}=\sqrt{\rho \mu} D / B$ (here $U$ and $B$ are respectively the root-meansquared velocity and dynamo-generated magnetic field in the fluid shell). Numerical models taken along this path can therefore be understood as continuously progressing from imperfect towards geophysically appropriate conditions in all relevant aspects of their inputs and outputs. The dimensional values of $\tau_{\Omega}, \tau_{U}$, and $\tau_{A}$ reached in our models and at the end of path are listed together with Earth's core estimates in Supplementary Table 1 (see ref. ${ }^{17}$ for a complete list of dimensionless time scale ratios achieved in the models).

Stratified core case. The Midpath-S model (Supplementary Table 1) explores the effects of a possible upper outer core stratification ${ }^{43}$ on the occurrence of simulated jerks. Within the Boussinesq approximation, stratification is modelled by adding an adverse density anomaly gradient ${ }^{44}$ to the background gradient prescribed by the neutral buoyancy conditions described above:

$$
\frac{\mathrm{d} C}{\mathrm{~d} r}=\frac{-N^{2} \rho}{2 g_{o}}\left(1+\tanh \left(\left(r-r_{s}\right) / \delta\right)\right)
$$

Here $N$ is the Brunt-Vaïsala frequency pertaining to the stratification level at the core surface, $r$ is radius, $r_{s}=3340 \mathrm{~km}$ is the radius at which stratification sets in, and $\delta=10^{-2} D=22.6 \mathrm{~km}$ is the thickness of the stratified layer front. The thickness of the stratified layer is $r_{o}-r_{s}=140 \mathrm{~km}$, as proposed in ref. ${ }^{43}$. In the Midpath-S model we set $N=1 / \tau_{\Omega}$, as also proposed in ref. ${ }^{43}$. The output of the Midpath-S model demonstrates the preservation of simulated jerks against core stratification, albeit at a reduced energy level given the modifications of the background magnetic field that guides the waves. 
Dimensional rescaling of dimensionless model output. The dimensionless model length unit is adjusted to the thickness $D=2260 \mathrm{~km}$ of Earth's core. Time is rescaled by adjusting the magnetic diffusion time scale $\tau_{\eta}=D^{2} / \eta$ to the value $\tau_{\eta}=135000 \mathrm{yr}$, corresponding to a value $\eta=1.2 \mathrm{~m}^{2} / \mathrm{s}$ at the midpoint of current estimates ${ }^{25}$. Given the invariance of the magnetic Reynolds number $R m=\tau_{\eta} / \tau_{U} \approx 1000$ along the parameter space path, this rescaling choice ensures $\tau_{U} \approx 130 \mathrm{yr}$ and Earth-like convective geomagnetic variations ${ }^{17}$. The fluid and Alfvén wave velocities are rescaled by using these length and time units. The magnetic field amplitude is presented by setting the Elsasser magnetic field unit $\sqrt{\rho \mu \eta \Omega}$ to the value $0.9 \mathrm{mT}$. Given the approximate invariance of the Elsasser number $B^{2} / \rho \mu \eta \Omega \approx 20$ along the path ${ }^{25}$, this amounts to setting the root-mean-squared field amplitude within the core to a value of about $4 \mathrm{mT}$, in agreement with Earth's core current estimate $^{13}$. Note that concerning the time and magnetic field units our choices slightly differ (by less than $5 \%$ ) from ref. ${ }^{17}$, as we adopt here the same units across all simulations. This change is done in order to obtain a consistent comparison between the original path models and those with a modified setup (Midpath-S,I,H, Supplementary Table 1) introduced in this study. Finally, the density anomaly rescaling used in Supplementary Movie 3 follows from the velocity rescaling and from adjustment of the dimensionless, time average convective power in the shell to an estimate ${ }^{25}$ $P=3 \mathrm{TW}$ of the geodynamo power.

Jerk energy definition and scaling. In Fig. 1c,d, we present the energy $E_{\mathrm{SA}}$ of the magnetic acceleration, defined as a mean-squared average over Earth's surface $S_{\mathrm{E}}$ :

$$
E_{\mathrm{SA}}=\left\langle\left(\partial_{t}^{2} \mathbf{B}\right)^{2}\right\rangle=\frac{1}{S_{\mathrm{E}}} \int_{S_{\mathrm{E}}}\left(\frac{\partial^{2} \mathbf{B}}{\partial t^{2}}\right)^{2} \mathrm{~d} S
$$


To facilitate comparison of the model output with geomagnetic field models of limited temporal resolution, the jerk energy $E_{\mathrm{J}}$ is defined as a sliding finite difference between consecutive 3-yr time windows rather than an instantaneous rate-of-change:

$$
E_{\mathrm{J}}(t)=\left\langle\left(\left[\partial_{t}^{2} \mathbf{B}\right]_{t}^{t+3 \mathrm{yr}}-\left[\partial_{t}^{2} \mathbf{B}\right]_{t-3 \mathrm{yr}}^{t}\right)^{2}\right\rangle .
$$

As introduced above, the angle brackets denote the average over Earth's surface, and the square brackets denote a time average. Jerk recurrence statistics in Fig. 4b are obtained from time series (Fig. 4a) of $E_{\mathrm{J}}$, by dividing the duration of the model run with the number of samples reaching or exceeding a given jerk energy. In Supplementary Fig. 2, jerk energies at 10, 30 and $100 \mathrm{yr}$ recurrence times are extracted from Fig. $4 \mathrm{~b}$ and scaled with the path parameter $\epsilon$, revaling a common dependency in $\epsilon^{-0.19 \pm 0.01}$. The end-of-path prediction in Fig. $4 \mathrm{~b}$ is obtained by collapsing the jerk statistics onto a single master curve according to this scaling, and extrapolating the master curve to the end-of-path conditions corresponding to $\epsilon=10^{-7}$.

The amplitude of a secular acceleration pulse scales with the magnetic field amplitude $B$ times the wave-induced flow acceleration $U / \tau_{A}$. Given that the pulse duration should also scale with $\tau_{A}$, jerk energy then scales with

$$
E_{\mathrm{J}} \sim \frac{\tau_{A}}{3 \mathrm{yr}}\left(B U / \tau_{A}\right)^{2} \sim \frac{(B U)^{2}}{\tau_{A}}
$$

Along the parameter space path, the dimensional values of $U$ and $B$ are approximately preserved, and the above scaling suggests that $E_{J}$ should be inversely proportional to $\tau_{A}$. The Alfvén number $A=\tau_{A} / \tau_{U}$ has been shown ${ }^{25}$ to scale like $\epsilon^{0.25}$ along the path. The invariance of $\tau_{U}$ along the path (Supplementary Table 1) then leads to $\tau_{A} \sim \epsilon^{0.25}$ and $E_{J} \sim \epsilon^{-0.25}$, close to the numerical result 
$E_{J} \sim \epsilon^{-0.19}$. The residual discrepancy mainly stems from the value of $B$ which slightly decreases along the path (see ref. ${ }^{25}$ ).

Identification of quasi-geostrophic Alfvén waves. In Supplementary Fig. 1 we repeat the analysis carried out in ref. ${ }^{17}$ to identify hydromagnetic wave propagation. The flow acceleration patterns that we analyse have a columnar structure that derives from the dominant rotational constraint of the Coriolis force. At any given time $t$, cylindrical radius $s$ and at a fixed analysis longitude $\varphi_{0}$, we therefore first compute the columnar average $\partial u_{c} / \partial t$ of azimuthal flow acceleration:

$$
\frac{\partial u_{c}}{\partial t}\left(s, \varphi_{0}, t\right)=\frac{1}{z_{+}-z_{-}} \int_{z_{-}}^{z_{+}} \frac{\partial\left(\mathbf{u} \cdot \mathbf{e}_{\varphi}\right)}{\partial t}\left(s, \varphi_{0}, z, t\right) \mathrm{d} z .
$$

Here $s, \varphi, z$ are cylindrical coordinates, $\mathbf{e}_{\varphi}$ is the unit vector in the azimuthal direction, and the vertical integral is evaluated between the lower and upper heights $z_{-,+}$of an axial column parallel to the rotation vector $\mathbf{\Omega}$ at cylindrical radius $s$. We then represent time-cylindrical radius maps of $\partial u_{c} / \partial t$ and overplot ray-tracing theoretical propagation tracks obtained by integrating in time the column-averaged Alfvén velocity $c_{A}$ and column-averaged cylindrical radial fluid velocity $V_{s}$ :

$$
\begin{aligned}
& c_{A}\left(s, \varphi_{0}, t\right)=\sqrt{\frac{1}{z_{+}-z_{-}} \int_{z_{-}}^{z_{+}} \frac{\left(\mathbf{B} \cdot \mathbf{e}_{s}\right)^{2}}{\rho \mu}\left(s, \varphi_{0}, z, t\right) \mathrm{d} z,} \\
& V_{s}\left(s, \varphi_{0}, t\right)=\frac{1}{z_{+}-z_{-}} \int_{z_{-}}^{z_{+}} \mathbf{u}\left(s, \varphi_{0}, z, t\right) \cdot \mathbf{e}_{s} \mathrm{~d} z .
\end{aligned}
$$

Here $\mathbf{e}_{s}$ is the unit vector in the cylindrical radial direction. The adherence of $\partial u_{c} / \partial t$ to the Alfvén tracks and deviation from material upwelling tracks demonstrates Alfvén wave propagation.

Length-of-day variations. The numerical simulation solves for the deviations $\Omega_{M}$ of the mantle angular velocity from the background planetary rotation rate $\Omega$ (see ref. ${ }^{42}$ for details):

$$
I_{\mathrm{M}} \frac{\mathrm{d} \Omega_{\mathrm{M}}}{\mathrm{d} t}=\Gamma_{\mathrm{M}}+\Gamma_{\mathrm{G}} .
$$


Here $I_{\mathrm{M}}$ is the Earth's mantle moment of inertia, and $\Gamma_{\mathrm{M}, \mathrm{G}}$ are respectively the magnetic and gravitational torques felt by the mantle. The corresponding rate of change $\mathrm{d}(\mathrm{LOD}) / \mathrm{d} t$ in the length of the day is then

$$
\frac{\mathrm{d}(\mathrm{LOD})}{\mathrm{d} t}=-\frac{2 \pi}{\Omega^{2}} \frac{\mathrm{d} \Omega_{\mathrm{M}}}{\mathrm{d} t}
$$

where we have used $\Omega_{\mathrm{M}} \ll \Omega$. Time series of $\mathrm{d}(\mathrm{LOD}) / \mathrm{d} t$ in the vicinity of jerk events are presented in Supplementary Fig. 4. The magnetic acceleration pulses cause pulses in $\mathrm{d}^{2} \Gamma_{\mathrm{M}} / \mathrm{d} t^{2}$, and hence rapid inflexions in $\mathrm{d}(\mathrm{LOD}) / \mathrm{d} t$ with shape similar to that observed in geodetic time series ${ }^{31,34}$. Note though that the amplitude of the inflexions is significantly weaker in the numerical simulations than in Earth's core, because the inverse squared Alfvén number $1 / A^{2}$ measuring the relative importance of magnetic forces and inertia is about 50 times weaker $^{25}$ in the Midpath model than in the core.

Numerical Implementation. Our numerical implementation involves a decomposition of the fields in spherical harmonics up to degree and order 133, and a discretisation in the radial direction on a second-order finite-differencing scheme (see ref. ${ }^{25}$ for numerical resolution details). We use the spherical harmonics transform library ${ }^{45}$ SHTns freely available at https://bitbucket.org /nschaeff/shtns. Time stepping is of second-order, semi-implicit type. Angular momentum conservation is controlled at each time step. To handle an increasing hydrodynamic turbulence along the path that does however only weakly affect the large-scale solution ${ }^{25}$, hyperdiffusion is implemented on the velocity and density anomaly fields, but not on the magnetic field which remains fully resolved. The details, physical justification and validation of this approximation are presented in ref. ${ }^{25}$. Each model on the path is initialised using the output of the previous step. Integration times after statistical equilibration are listed in Supplementary Table 1. In our main 
models these represent at least $18 \%$ of a magnetic diffusion time and $75 \%$ of a dipole decay time

$r_{o}^{2} / \pi^{2} \eta$. Within this time all model outputs are in a statistically-steady state ${ }^{17}$ demonstrating selfsustained dynamo action. In particular, all models produced an axial dipole-dominated magnetic field that did not reverse polarity.

Data availability. The numerical code and the simulation datasets analysed during the current study are available from the corresponding author on reasonable request. 
1. Brown, W., Mound, J. \& Livermore, P. Jerks abound: An analysis of geomagnetic observatory data from 1957 to 2008. Phys. Earth Planet. Int. 223, 62 - 76 (2013).

2. Courtillot, V., Ducruix, J. \& Le Mouël, J.-L. Sur une accélération récente de la variation séculaire du champ magnétique terrestre. C.R. Acad. Sci. Paris. D 287, 1095-1098 (1978).

3. Malin, S. R. C., Hodder, B. M. \& Barraclough, D. R. Geomagnetic secular variation: A jerk in 1970. In Cardús, J. O. (ed.) Scientific Contributions in Commemoration of Ebro Observatory’s 75th Anniversary (1983).

4. Lesur, V., Wardinski, I., Hamoudi, M. \& Rother, M. The second generation of the GFZ Reference Internal Magnetic Model: GRIMM-2. Earth Planets Space 62, 765-773 (2010).

5. Finlay, C. C., Olsen, N., Kotsiaros, S., Gillet, N. \& Tøffner-Clausen, L. Recent geomagnetic secular variation from Swarm and ground observatories as estimated in the CHAOS-6 geomagnetic field model. Earth, Planets and Space 68, 112 (2016).

6. Chulliat, A., Thebault, E. \& Hulot, G. Core field acceleration pulse as a common cause of the 2003 and 2007 geomagnetic jerks. Geophys. Res. Lett. 37 (2010).

7. Chulliat, A. \& Maus, S. Geomagnetic secular acceleration, jerks, and a localized standing wave at the core surface from 2000 to 2010. J. Geophys. Res. 119, 1531-1543 (2014).

8. Chulliat, A., Alken, P. \& Maus, S. Fast equatorial waves propagating at the top of the Earth's core. Geophys. Res. Lett. 42, 3321-3329 (2015). 
9. Torta, J. M., Pavón-Carrasco, F. J., Marsal, S. \& Finlay, C. C. Evidence for a new geomagnetic jerk in 2014. Geophys. Res. Lett. 42, 7933-7940 (2015).

10. Pais, M. A., Alberto, P. \& Pinheiro, F. J. G. Time-correlated patterns from spherical harmonic expansions: Application to geomagnetism. J. Geophys. Res. 120, 8012-8030 (2015).

11. Braginsky, S. I. Short-period geomagnetic secular variation. Geophys. Astrophys. Fluid Dyn. 30, 1-78 (1984).

12. Bloxham, J., Zatman, S. \& Dumberry, M. The origin of geomagnetic jerks. Nature 420, 65-68 (2002).

13. Gillet, N., Jault, D., Canet, E. \& Fournier, A. Fast torsional waves and strong magnetic field within the Earth's core. Nature 465, 74-77 (2010).

14. Wicht, J. \& Christensen, U. R. Torsional oscillations in dynamo simulations. Geophys. J. Int. 181, 1367-1380 (2010).

15. Teed, R. J., Jones, C. A. \& Tobias, S. M. The dynamics and excitation of torsional waves in geodynamo simulations. Geophys. J. Int. 196, 724-735 (2014).

16. Schaeffer, N., Jault, D., Nataf, H.-C. \& Fournier, A. Turbulent geodynamo simulations: a leap towards Earth's core. Geophys. J. Int. 211, 1-29 (2017).

17. Aubert, J. Geomagnetic acceleration and rapid hydromagnetic wave dynamics in advanced numerical simulations of the geodynamo. Geophys. J. Int. (2018, in press). Preprint available at http://www.arxiv.org/abs/1804.04865. 
18. Cox, G., Livermore, P. \& Mound, J. The observational signature of modelled torsional waves and comparison to geomagnetic jerks. Phys. Earth Planet. Int. 255, 50 - 65 (2016).

19. Wardinski, I., Holme, R., Asari, S. \& Mandea, M. The 2003 geomagnetic jerk and its relation to the core surface flows. Earth Plan. Sci. Lett. 267, 468 - 481 (2008).

20. Silva, L. \& Hulot, G. Investigating the 2003 geomagnetic jerk by simultaneous inversion of the secular variation and acceleration for both the core flow and its acceleration. Phys. Earth Planet. Int. 198-199, 28 - 50 (2012).

21. Gillet, N., Jault, D. \& Finlay, C. C. Planetary gyre, time-dependent eddies, torsional waves and equatorial jets at the Earth's core surface. J. Geophys. Res. 120, 3991-4013 (2015).

22. Christensen, U. R., Aubert, J. \& Hulot, G. Conditions for Earth-like geodynamo models. Earth. Plan. Sci. Let. 296, 487-496 (2010).

23. Mound, J., Davies, C. \& Silva, L. Inner core translation and the hemispheric balance of the geomagnetic field. Earth Plan. Sci. Lett. 424, 148 - 157 (2015).

24. Aubert, J., Finlay, C. C. \& Fournier, A. Bottom-up control of geomagnetic secular variation by the Earth's inner core. Nature 502, 219-223 (2013).

25. Aubert, J., Gastine, T. \& Fournier, A. Spherical convective dynamos in the rapidly rotating asymptotic regime. J. Fluid. Mech. 813, 558-593 (2017).

26. Yadav, R. K., Gastine, T., Christensen, U. R., Wolk, S. J. \& Poppenhaeger, K. Approaching a realistic force balance in geodynamo simulations. PNAS 113, 12065-12070 (2016). 
27. Sheyko, A., Finlay, C. C. \& Jackson, A. Magnetic reversals from planetary dynamo waves. Nature 539, 551-554 (2016).

28. Labbé, F., Jault, D. \& Gillet, N. On magnetostrophic inertia-less waves in quasi-geostrophic models of planetary cores. Geophys. Astrophys. Fluid Dyn. 109, 587-610 (2015).

29. Bardsley, O. P. \& Davidson, P. A. Inertial-Alfvén waves as columnar helices in planetary cores. J. Fluid. Mech. 805 (2016).

30. Dormy, E. \& Mandea, M. Tracking geomagnetic impulses at the core-mantle boundary. Earth Plan. Sci. Lett. 237, 300 - 309 (2005).

31. Mandea, M. et al. Geomagnetic Jerks: Rapid Core Field Variations and Core Dynamics. Space. Sci. Rev. 155, 147-175 (2010).

32. Dean, R. G. \& Dalrymple, R. A. Water wave mechanics for engineers and scientists, vol. 2 (World Scientific Publishing Company, 1991).

33. Olsen, N. \& Mandea, M. Rapidly changing flows in the Earth's core. Nature Geosci. 1, 390-394 (2008).

34. Holme, R. \& de Viron, O. Geomagnetic jerks and a high-resolution length-of-day profile for core studies. Geophys. J. Int. 160, 435-439 (2005).

35. Lesur, V., Whaler, K. \& Wardinski, I. Are geomagnetic data consistent with stably stratified flow at the core-mantle boundary? Geophys. J. Int. 201, 929-946 (2015). 
36. Schaeffer, N. \& Jault, D. Electrical conductivity of the lowermost mantle explains absorption of core torsional waves at the equator. Geophys. Res. Lett. 43, 4922-4928 (2016).

37. Holme, R. \& de Viron, O. Characterization and implications of intradecadal variations in length of day. Nature 499, 202-204 (2013).

38. Fournier, A. et al. An Introduction to Data Assimilation and Predictability in Geomagnetism. Space. Sci. Rev. 155, 247-291 (2010).

39. Aubert, J. Geomagnetic forecasts driven by thermal wind dynamics in the Earth's core. Geophys. J. Int. 203, 1738-1751 (2015).

40. Fournier, A., Aubert, J. \& Thébault, E. A candidate secular variation model for IGRF-12 based on Swarm data and inverse geodynamo modelling. Earth. Planets. Space. 67, 81 (2015).

41. Thébault, E. et al. International geomagnetic reference field: the twelfth generation. Earth. Planets. Space. 67, 79 (2015).

42. Pichon, G., Aubert, J. \& Fournier, A. Coupled dynamics of Earth's geomagnetic westward drift and inner core super-rotation. Earth Planet. Sci. Lett. 437, 114-126 (2016).

43. Buffett, B. A. Geomagnetic fluctuations reveal stable stratification at the top of the Earth's core. Nature 507, 484-487 (2014).

44. Nakagawa, T. Effect of a stably stratified layer near the outer boundary in numerical simulations of a magnetohydrodynamic dynamo in a rotating spherical shell and its implications for Earth's core. Phys. Earth. Planet. Int. 187, 342 - 352 (2011). 
45. Schaeffer, N. Efficient spherical harmonic transforms aimed at pseudospectral numerical simulations. Geophys. Geochem. Geosystems. 14, 751-758 (2013). 
Acknowledgements JA acknowledges support from French Programme National de Planétologie (PNP) of CNRS/INSU, and from the Fondation Del Duca of Institut de France. This work was granted access to the HPC resources of S-CAPAD, IPGP, France, and to the HPC resources of IDRIS and CINES under the allocation 2016-A0020402122 made by GENCI. The results presented in this work rely on data collected at magnetic observatories. The authors thank the national institutes that support them and INTERMAGNET for promoting high standards of magnetic observatory practice (www.intermagnet.org).

Author contributions J.A. designed the project, designed and carried out the numerical experiments, and wrote the manuscript. C.C.F. processed the geomagnetic data, constructed the CHAOS-6x5 geomagnetic field model and led its comparison with the simulation results. J.A. and C.C.F. processed the results and discussed the manuscript.

Author Information Correspondence and requests for materials should be addressed to J. Aubert (email: aubert@ipgp.fr). The authors declare no competing financial interests. 
Figure 1 Signature of geomagnetic jerks at Earth's surface. a,c,e, Observatory geomagnetic data and output from the CHAOS-6x5 geomagnetic field model ${ }^{5}$ based on satellite and ground observations. b,d,f, Output from the Midpath numerical model (Methods and Supplementary Table 1). a, Time series of the downward geomagnetic secular variation (rate of change of the geomagnetic field, blue lines and crosses) from annual differences of revised monthly means at two selected observatories, Kourou and Tamanrasset (marked as green dots in e), plotted together with the CHAOS-6x5 output (black). b, Downward vertical secular variation time series in the Midpath numerical model, at two locations marked with green dots in $\mathbf{f}$. c,d, Time series of the mean-squared secular acceleration $E_{\mathrm{SA}}$ (grey) and of jerk energy $E_{\mathrm{J}}$ (black, see Methods for definitions and Fig. 4a for a longer time series of $E_{\mathrm{J}}$ ), showing how secular acceleration pulses relate to the strong acceleration changes that characterise jerks. As identified locally from secular variation time series and globally by peaks in $E_{\mathrm{J}}$, vertical dashed lines in a,c mark geomagnetic jerks ${ }^{6-9}$ occurring near epochs 2007.5, 2011, 2014 and in $\mathbf{b}, \mathbf{d}$ the synthetic jerk event used to define the simulation time origin. $\mathbf{e , f}$, Hammer projections of the radial secular acceleration (orange is outwards) before and after a jerk event, showing patterns alternating in time over a large portion of Earth's surface.

Figure 2 Signature of geomagnetic jerks at Earth's core surface. a,b, Output from the CHAOS-6x5 geomagnetic field model ${ }^{5}$. c-f, Output from the Midpath numerical dynamo model. a,c, Time-longitude plots of the radial secular acceleration at the equator (orange is outwards), filtered at spherical harmonic degree 9. The horizontal dashed lines locate geomagnetic jerks epochs ${ }^{6-9}$ in $\mathbf{a}$ and the synthetic jerk time in $\mathbf{c}$. The vertical lines respectively 
locate the longitudes selected for analysis in ref. ${ }^{5}$ for the real events, and in $\mathbf{f}$ for the synthetic event. b,d, Hammer projections of the radial secular acceleration (same spatial filtering as in a,c) before and after jerk events, showing localised patterns alternating in time (see also a numerical model temporal sequence in Supplementary Movie 1). Black curves again locate the longitudes selected for analysis. e, Miller map showing details of the azimuthal flow acceleration at the core surface (native model spatial resolution, blue is westwards) during the simulated jerk event (see also global map and temporal sequence in Supplementary Movie 2). The black vertical lines locate the analysis longitude used in f. f, Temporal evolution of the core surface, equatorial azimuthal flow acceleration at the analysis longitude, showing the structure of the wave packet that causes the jerk.

\section{Figure 3 Hydromagnetic waves inside the core and magnetic field structure from the}

Midpath model. a, Planform of the azimuthal flow acceleration (blue is westward) at time -3.22 yr before the jerk event, in a quarter of the equatorial plane between longitudes $0^{\circ} E$ and $90^{\circ} \mathrm{E}$. Also shown are the directions of the rotation vector $\mathbf{\Omega}$ and the wave vector $\mathbf{k}$. $\mathbf{b}$, Meridional planform of azimuthal flow acceleration outside the axial cylinder tangent to Earth's core and at the analysis longitude located by a black line in a. See supplementary Movie 4 for the corresponding temporal sequences. c, closeup of equatorial azimuthal flow acceleration corresponding to the dashed box in a, showing the concentrated wave structures below the core-mantle boundary. d, Semi-transparent detail of a (see second dashed box), with a volumetric rendering of the magnetic field lines (grey, thickness proportional to local magnetic field amplitude) that channel and focus the waves towards the core surface. e, Hammer projection 
of the radial magnetic field at the core surface (native model spatial resolution, orange is outwards) at time -3.22 yr. Arrows in d,e locate the core surface magnetic flux patches where the focused waves emerge.

Figure 4 Statistics of jerk recurrence time. a, Time series of jerk energy $E_{\mathrm{J}}$ (see Methods) in the $29 \%$ of path and Midpath models (Supplementary Table 1). The arrow locates the event at time $0 \mathrm{yr}$ analysed in Figs. 1-3. b, Distribution of the average recurrence time of jerks reaching or exceeding a given energy, for models within the rapid rotation regime ${ }^{17}$ (solid lines). Also shown is an extrapolation (see Methods and Supplementary Fig. 2) of the recurrence time distribution for the Earth's core conditions (dashed line and light grey shaded uncertainty area). The dark grey line segment locates the output of CHAOS-6x5 as estimated from Fig. 1c (three jerks with $E_{\mathrm{J}} \geq 80 \mathrm{nT}^{2} . \mathrm{yr}^{-4}$ and two with $E_{\mathrm{J}} \geq 90 \mathrm{nT}^{2} \cdot \mathrm{yr}^{-4}$ within 19 years). 

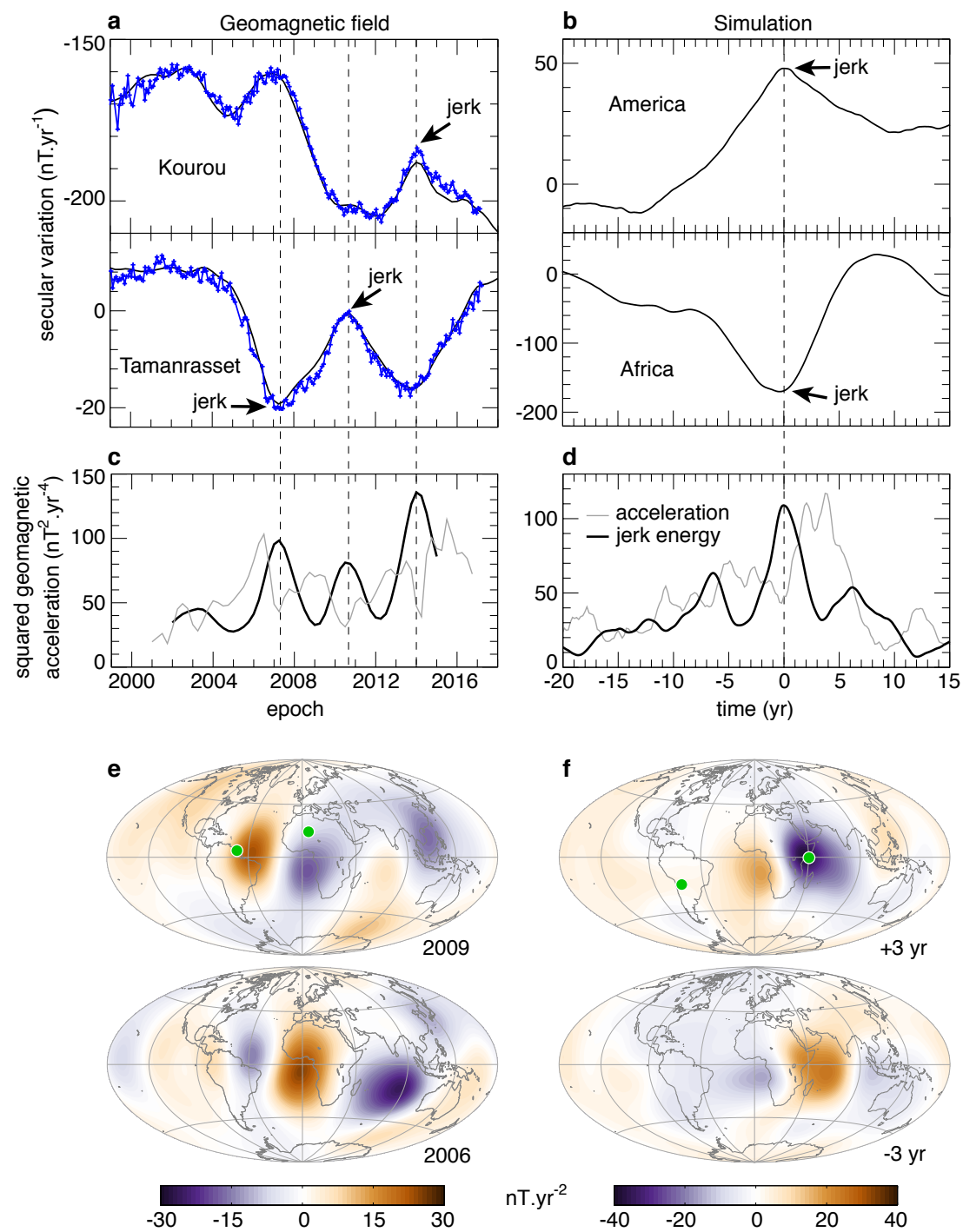

Figure 1 



Figure 2 

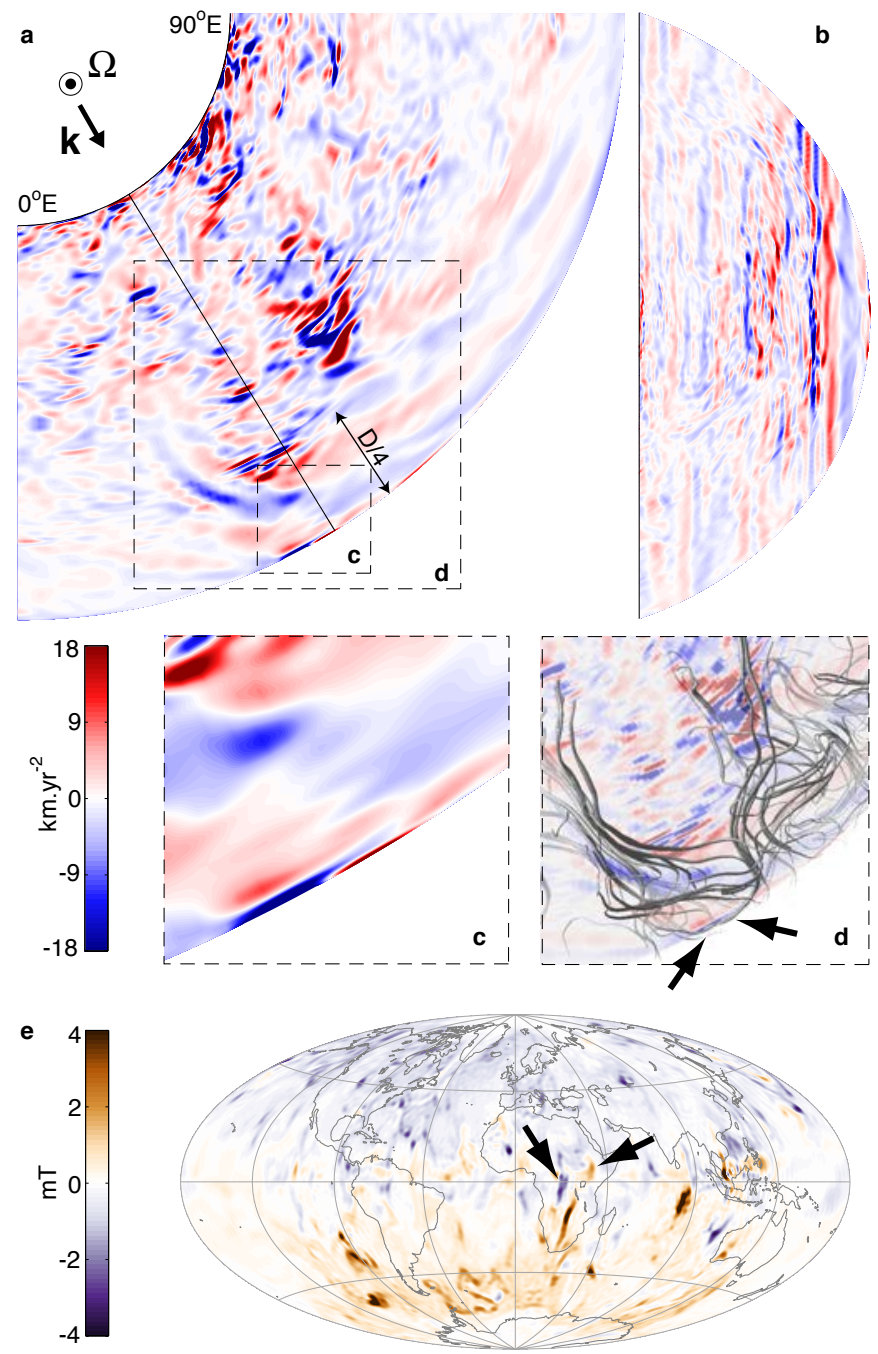

Figure 3 

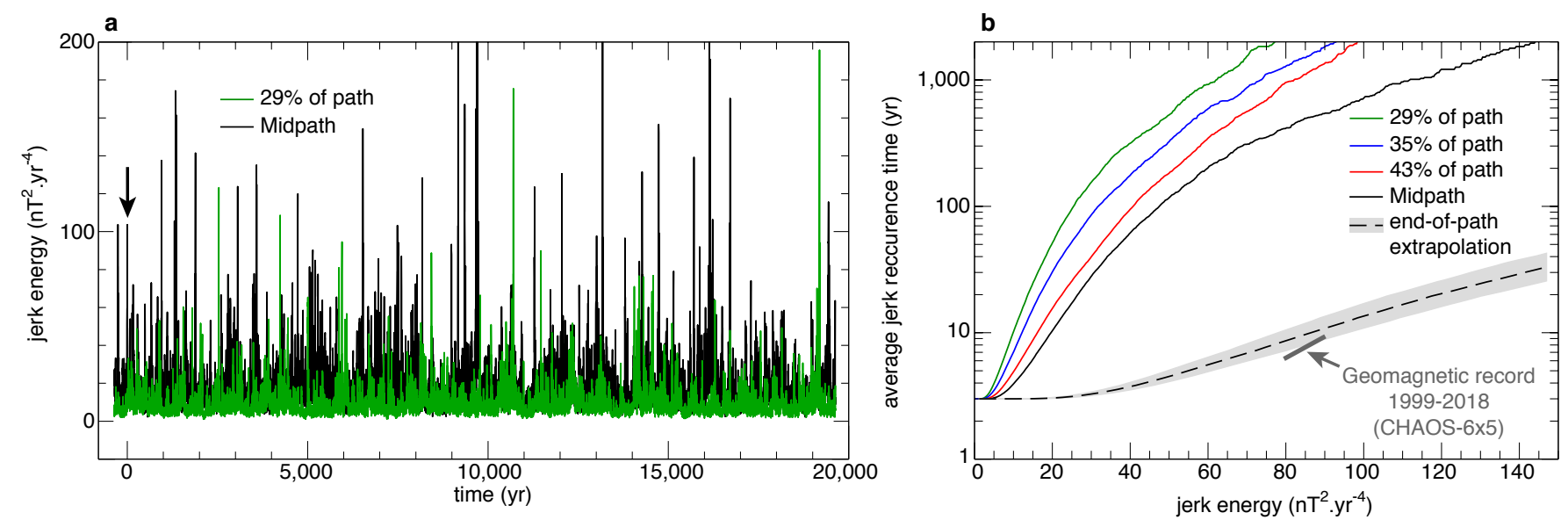

Figure 4 


\section{Supplementary information for: Geomagnetic jerks and rapid hydromagnetic waves focusing at Earth's core surface}

Julien Aubert ${ }^{1}$ and Christopher C. Finlay ${ }^{2}$

${ }^{1}$ Institut de Physique du Globe de Paris, Sorbonne Paris Cité, Université Paris Diderot, CNRS

UMR 7154 CNRS, F-75005 Paris, France.

${ }^{2}$ Division of Geomagnetism, National Space Institute, Technical University of Denmark, Elektrovej, 2800 Kgs. Lyngby, Denmark

\section{Contents:}

Supplementary Table 1

Supplementary Figs. 1-4

Captions for Supplementary Movies 1-4 


\begin{tabular}{|c|c|c|c|c|c|c|c|c|}
\hline Label & $\begin{array}{l}\text { Path } \\
\text { parameter } \epsilon\end{array}$ & $\begin{array}{l}\text { Path } \\
\text { position }\end{array}$ & $\frac{\Delta \sigma_{m}}{D \sigma_{c}}$ & $\begin{array}{l}\text { core } \\
\text { surface } \\
\text { buoyancy }\end{array}$ & $\tau_{U}(\mathrm{yr})$ & $\tau_{A}(\mathrm{yr})$ & $2 \pi \tau_{\Omega}(\mathrm{yr})$ & $\begin{array}{l}\text { Integration } \\
\text { time (yr) }\end{array}$ \\
\hline & $10^{-2}$ & $29 \%$ & $10^{-4}$ & neutral & 129 & 31.5 & 1.0 & 42900 \\
\hline & $3.3310^{-3}$ & $36 \%$ & $10^{-4}$ & neutral & 126 & 24.0 & 0.6 & 34600 \\
\hline & $10^{-3}$ & $43 \%$ & $10^{-4}$ & neutral & 123 & 18.2 & 0.3 & 24900 \\
\hline Midpath & $3.3310^{-4}$ & $50 \%$ & $10^{-4}$ & neutral & 125 & 14.3 & 0.2 & 24400 \\
\hline Midpath-I & $3.3310^{-4}$ & $50 \%$ & 0 & neutral & 120 & 14.2 & 0.2 & 11400 \\
\hline Midpath-H & $3.3310^{-4}$ & $50 \%$ & $10^{-3}$ & neutral & 128 & 14.6 & 0.2 & 11300 \\
\hline Midpath-S & $3.3310^{-4}$ & $50 \%$ & $10^{-4}$ & adverse & 121 & 14.5 & 0.2 & 10100 \\
\hline End of path & $10^{-7}$ & $100 \%$ & & & 130 & 1.9 & $3.210^{-3}$ & \\
\hline Earth & & & & & $\approx 140$ & $\approx 2$ & $2.710^{-3}$ & \\
\hline
\end{tabular}

Supplementary Table 1: Models along a parameter space path to Earth's core. Key parameters and corresponding dimensional time scale values for numerical models located along a parameter space path ${ }^{25}$ towards Earth's core conditions. See Methods for definitions and ref. ${ }^{17}$ for complete parameter data. Dimensional time scales values are obtained from the dimensionless time scale ratios reported in ref. ${ }^{17}$ and the magnetic diffusion time scale set to $\tau_{\eta}=135000 \mathrm{yr}$ in this study. Also shown are the values, closely approaching Earth's core estimates, obtained by extrapolating scaling laws determined along the path ${ }^{25}$ to its end point. 

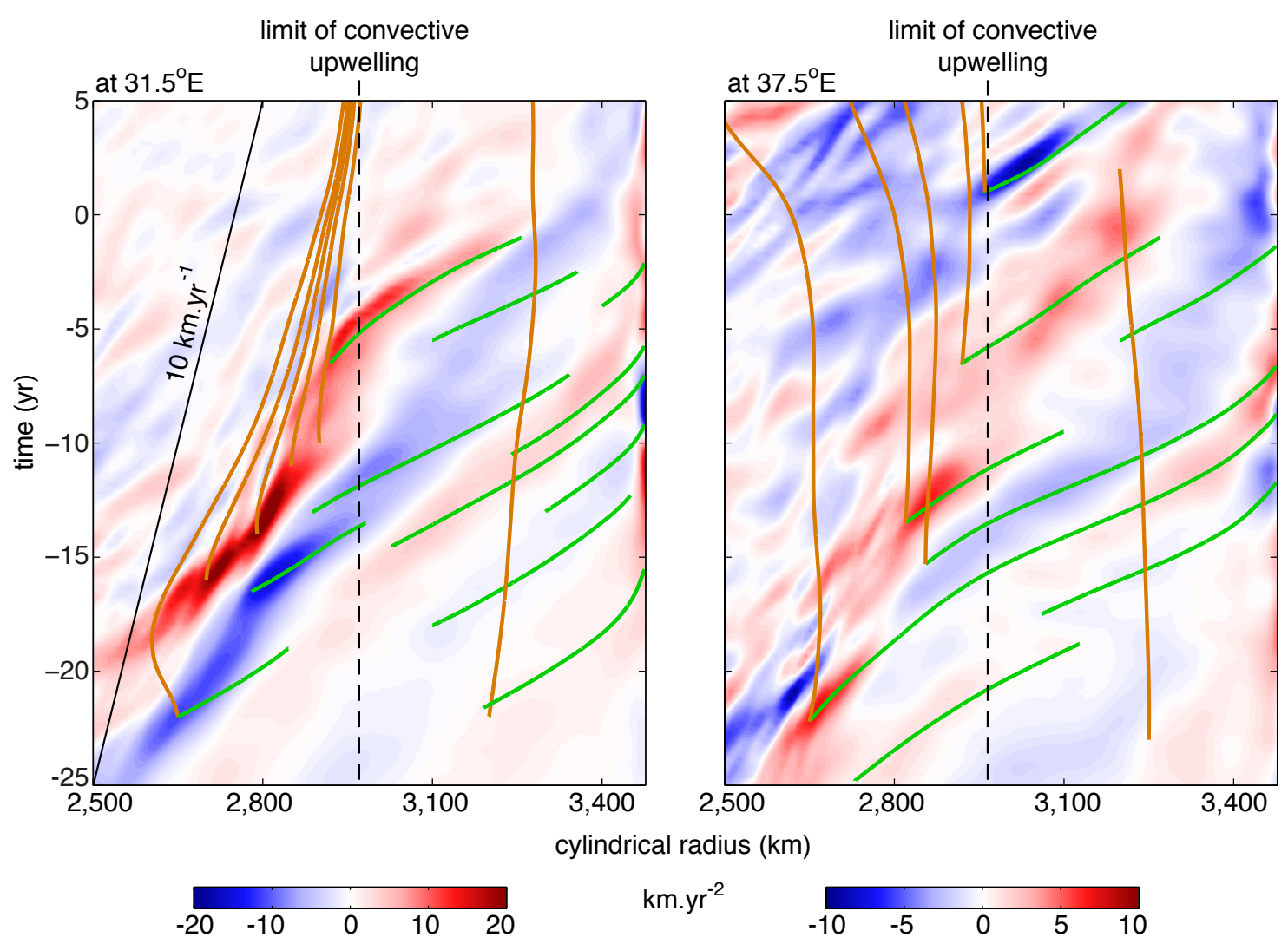

Supplementary Figure 1: Alfvén wave propagation in the upper outer core. Time-cylindrical radius diagrams of the column-averaged azimuthal flow acceleration $\partial u_{c} / \partial t$ (blue is westwards, see Methods for definitions) evaluated at two analysis longitudes, $31.5^{\circ} \mathrm{E}$ (as in Fig. $3 \mathrm{~b}$ ) and $37.5^{\circ} \mathrm{E}$. Similar to ref. ${ }^{17}$, green and brown curves respectively represent the ray-tracing theoretical propagation tracks of hydromagnetic waves at the column-averaged Alfvén speed $c_{A}$, and of material advection at the column-averaged cylindrical radial fluid velocity $V_{s}$. The slanted black line on the left panel denotes upward propagation at a speed $10 \mathrm{~km} / \mathrm{yr}$. 


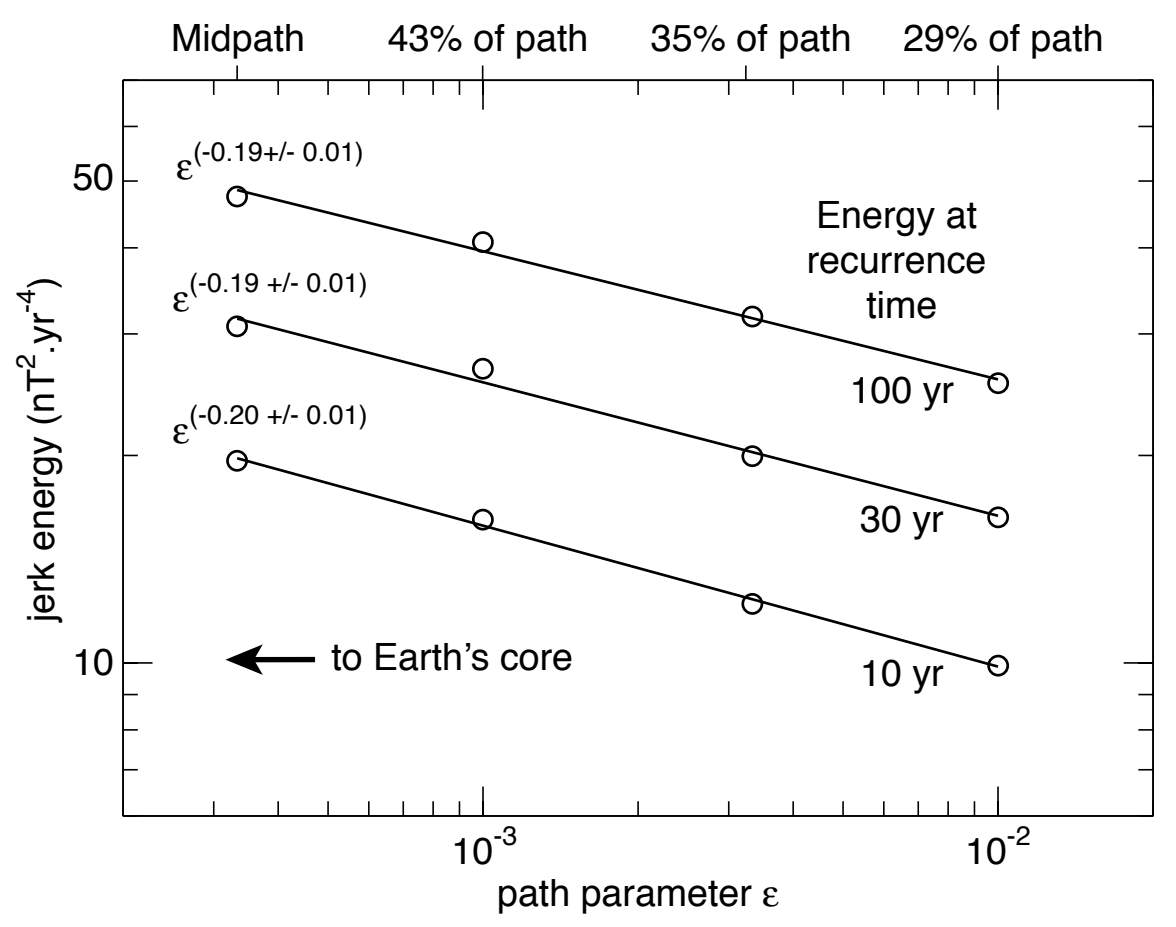

Supplementary Figure 2: Scaling of jerk energy along the parameter space path. Evolution of jerk energy at recurrence times 10,30 and 100 years, as extracted from Fig. 4b, with the path parameter $\epsilon$ (Methods and Supplementary Table 1). 


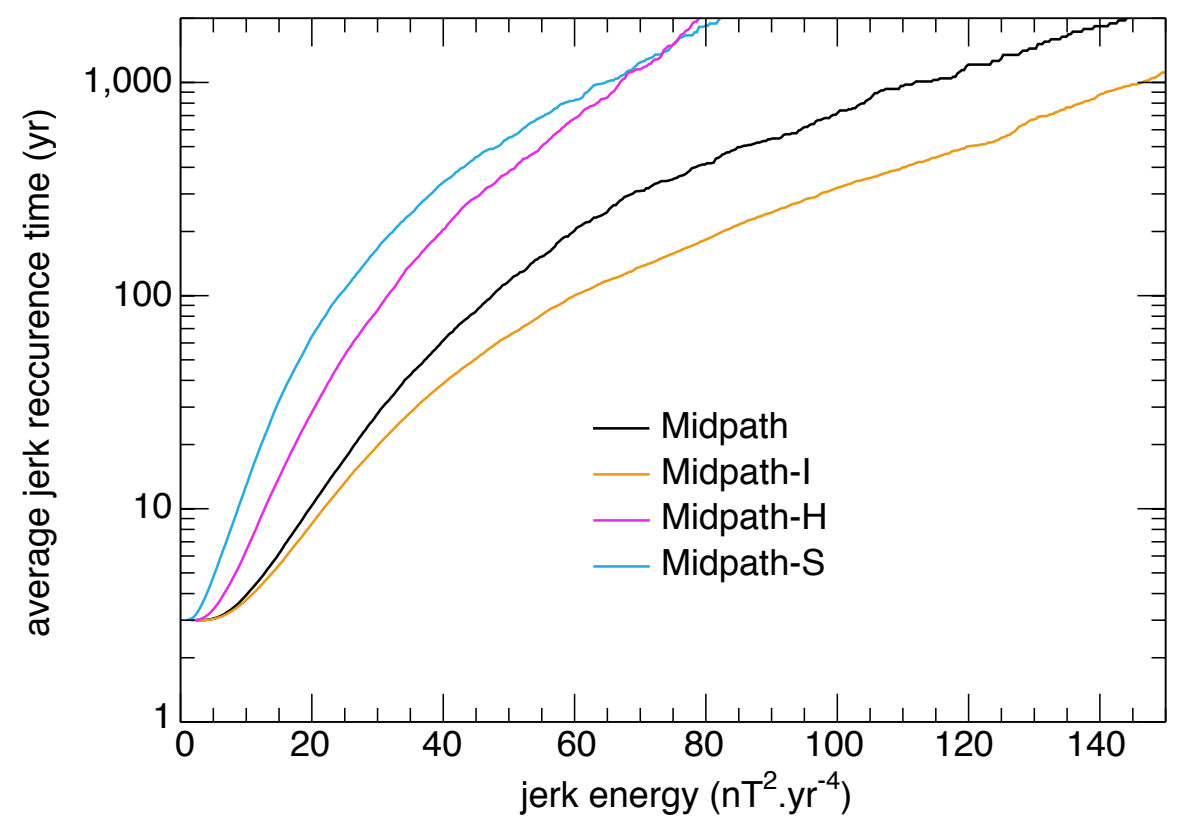

Supplementary Figure 3: Sensitivity of the jerk recurrence time distribution to physical conditions in the lower mantle and upper outer core. Distribution of the average recurrence time of jerks reaching or exceeding a given energy (same as Fig. 4b), for the models Midpath-I and Midpath-H with variable lower mantle electrical conductance, and model Midpath-S with a stratified region in the upper outer core (Methods). The Midpath model result from Fig. 4b is also reproduced for reference. 

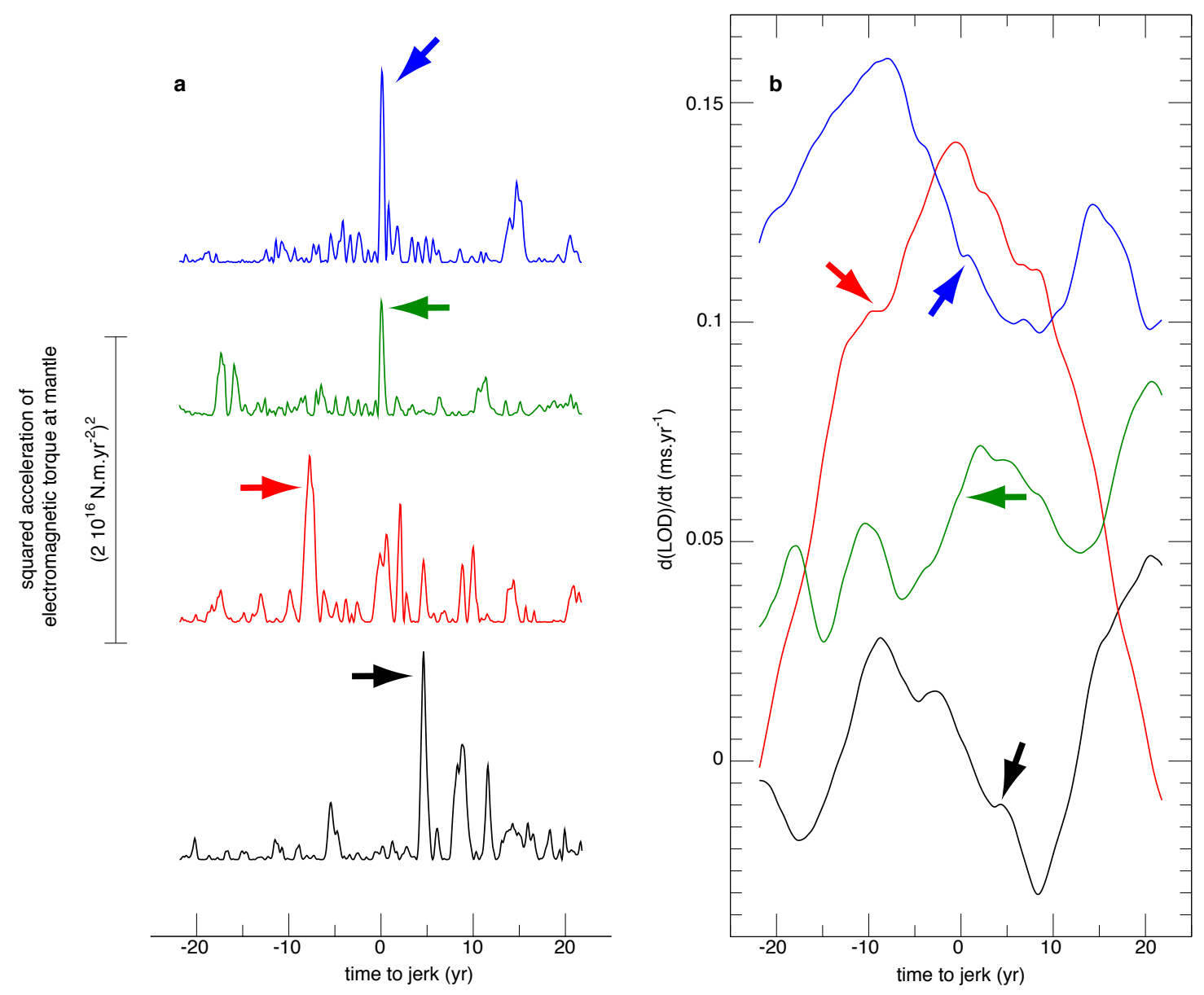

Supplementary Figure 4: Signature of simulated jerks in the length of the day. a, Squared acceleration $\left(\mathrm{d}^{2} \Gamma_{\mathrm{M}} / \mathrm{d} t^{2}\right)^{2}$ (see Methods for definitions) of the electromagnetic torque exerted on the mantle by the outer core, as a function of time in the vicinity of four jerk events of model Midpath-H. b, first time derivative $\mathrm{d}(\mathrm{LOD}) / \mathrm{d} t$ of the simulated length of the day, as a function of time during the same jerk events. Arrows locate the pulses in the torque accelerations, that correspond to rapid inflexions in the rate of change of the length-of-day. 
Supplementary Movie 1: Hammer projection of the core surface radial secular geomagnetic acceleration (orange is outwards) from the Midpath model, filtered at spherical harmonic degree 9 , in the vicinity of the jerk event occurring at time $0 \mathrm{yr}$.

Supplementary Movie 2: Hammer projection of the core surface azimuthal flow acceleration (blue is westwards) from the Midpath model, in the vicinity of the jerk event occurring at time $0 \mathrm{yr}$.

Supplementary Movie 3: Partial equatorial cut (left) and meridional cut outside the tangent cylinder (right) of the convective density anomaly (orange denotes lighter fluid) from the Midpath model in the vicinity of the jerk event occurring at time $0 \mathrm{yr}$. The meridional cut in the right panel is taken at the analysis longitude marked by a black line in the left panel.

Supplementary Movie 4: Partial equatorial cut (left) and meridional cut outside the tangent cylinder (right) of azimuthal flow acceleration (blue is westwards) from the Midpath model in the vicinity of the jerk event occurring at time $0 \mathrm{yr}$. The meridional cut in the right panel is taken at the analysis longitude marked by a black line in the left panel. 